\title{
Combined treatment with CBP and BET inhibitors reverses inadvertent activation of detrimental super enhancer programs in DIPG cells
}

Maria Wiese', Feda H. Hamdan², Klaudia Kubiak, Christopher Diederichs ${ }^{1,8}$, Gerrit H. Gielen ${ }^{3}$, Gunther Nussbaumer ${ }^{4}$, Angel M. Carcaboso $\mathbb{1 0}^{5}$, Esther Hulleman ${ }^{6,7}$, Steven A. Johnsen $\mathbb{1}^{2}$ and Christof M. Kramm ${ }^{1}$

\begin{abstract}
Diffuse intrinsic pontine gliomas (DIPG) are the most aggressive brain tumors in children with 5-year survival rates of only $2 \%$. About $85 \%$ of all DIPG are characterized by a lysine-to-methionine substitution in histone 3, which leads to global H3K27 hypomethylation accompanied by H3K27 hyperacetylation. Hyperacetylation in DIPG favors the action of the Bromodomain and Extra-Terminal (BET) protein BRD4, and leads to the reprogramming of the enhancer landscape contributing to the activation of DIPG super enhancer-driven oncogenes. The activity of the acetyltransferase CREBbinding protein (CBP) is enhanced by BRD4 and associated with acetylation of nucleosomes at super enhancers (SE). In addition, CBP contributes to transcriptional activation through its function as a scaffold and protein bridge. Monotherapy with either a CBP (ICG-001) or BET inhibitor (JQ1) led to the reduction of tumor-related characteristics. Interestingly, combined treatment induced strong cytotoxic effects in H3.3K27M-mutated DIPG cell lines. RNA sequencing and chromatin immunoprecipitation revealed that these effects were caused by the inactivation of DIPG SE-controlled tumor-related genes. However, single treatment with ICG-001 or JQ1, respectively, led to activation of a subgroup of detrimental super enhancers. Combinatorial treatment reversed the inadvertent activation of these super enhancers and rescued the effect of ICG-001 and JQ1 single treatment on enhancer-driven oncogenes in H3K27Mmutated DIPG, but not in H3 wild-type pedHGG cells. In conclusion, combinatorial treatment with CBP and BET inhibitors is highly efficient in H3K27M-mutant DIPG due to reversal of inadvertent activation of detrimental SE programs in comparison with monotherapy.
\end{abstract}

\section{Introduction}

Diffuse intrinsic pontine gliomas (DIPG) are pediatric high-grade gliomas (pedHGG) accounting for approximately $10-15 \%$ of pediatric central nervous system tumors. Despite major improvements in treatment

Correspondence: Maria Wiese (maria.wiese@med.uni-goettingen.de) ${ }^{1}$ Division of Pediatric Hematology and Oncology, Department of Child and Adolescent Health, University Medical Center Goettingen, Robert Koch Straße 40, Goettingen, Germany

${ }^{2}$ Gene Regulatory Mechanisms and Molecular Epigenetics Lab, Division of Gastroenterology and Hepatology, Mayo Clinic, 200 First Street SW, Rochester MN 55905, USA

Full list of author information is available at the end of the article These authors contributed equally: Maria Wiese, Feda H. Hamdan Edited by M. Agostini strategies, the prognosis for DIPG patients persists to be dismal with 5 -year survival rates of $2 \%$. Approximately $85 \%$ of all DIPGs are characterized by a mutation in the genes encoding either histone 3.3 or 3.1 , resulting in a lysine-to-methionine substitution at position 27 $(\mathrm{H} 3 \mathrm{~K} 27 \mathrm{M})^{1}$. The H3K27M mutation is supposed to contribute to this aggressive tumor biology, probably by inducing a stem cell-like phenotype ${ }^{2}$.

The H3K27M mutation significantly impairs EZH2 methyltransferase function within the PRC2 complex, and leads to dramatic chromatin changes characterized by a global loss of trimethylation accompanied by hyperacetylation at lysine 27 of histone 3 (H3K27me3) ${ }^{3}$. Acetylation at lysine 27 of histone 3 (H3K27ac) is mediated by

\section{(c) The Author(s) 2020}

(c) (i) Open Access This article is licensed under a Creative Commons Attribution 4.0 International License, which permits use, sharing, adaptation, distribution and reproduction cc) in any medium or format, as long as you give appropriate credit to the original author(s) and the source, provide a link to the Creative Commons license, and indicate if changes were made. The images or other third party material in this article are included in the article's Creative Commons license, unless indicated otherwise in a credit line to the material. If material is not included in the article's Creative Commons license and your intended use is not permitted by statutory regulation or exceeds the permitted use, you will need to obtain permission directly from the copyright holder. To view a copy of this license, visit http://creativecommons.org/licenses/by/4.0/. 
the KAT3 family of acetyltransferases (HATs) including CREB-binding protein (CBP). In addition to its function as an acetyltransferase, CBP serves as a protein bridge that connects other transcription factors to the transcription machinery, and acts as scaffold protein during the formation of multicomponent transcriptional regulatory complexes $^{4}$. CBP is necessary for the recruitment of diverse transcription factors, including the Bromodomain and Extra-Terminal (BET) protein BRD4, to genomic regions displaying an enrichment of acetylated nucleosomes, thereby promoting transcription ${ }^{4-6}$. Consequently, BRD4 and CBP are promising pharmaceutical targets for treatment of DIPG and pedHGG ${ }^{5,6}$.

Notably, BET inhibition has been reported to attenuate activation of oncogenic programs in DIPG driven by a subtype of enhancers referred to as "super enhancers" $(\mathrm{SE})^{7}$. SE are clusters of enhancers that are highly enriched for activation factors such as BRD4 and CBP, and are known to drive oncogenic programs in various malignancies ${ }^{8}$. In this study, we aim to evaluate the potential utility of combinatorial BET and CBP inhibition in DIPG. Furthermore, we characterized the effects of these inhibitors on SE programs.

\section{Materials and methods}

\section{Cell culture}

DIPG and pedHGG cell lines were cultured as previously described ${ }^{9}$. SF188 cells were cultured in DMEM/ F12. To provide various cellular phenotypes that represent the heterogeneity of DIPG tumor samples and may differentially respond to drug treatment, cells were grown as gliomaspheres in tumor stem cell medium (TSM) or under differentiation conditions in TSM supplemented with $10 \%$ FCS, as recommended in ref. ${ }^{9}$. Unless stated otherwise, cells were treated with $2.5 \mu \mathrm{M}$ ICG-001 (Calbiochem, Darmstadt, Germany), $2.5 \mu \mathrm{M}$ PRI-724 (Selleckchem, Munich, Germany), and $0.25 \mu \mathrm{M}(+) /-\mathrm{JQ} 1$ (Selleckchem) dissolved in DMSO.

\section{Cell viability assays}

MTT assays, to monitor $x$-fold cell growth over time, were carried out as described previously ${ }^{6}$. BrDU assays were conducted using the Cell Proliferation ELISA, BrDU-Kit (Merck, Darmstadt, Germany) according to the manufacturer's instructions. Crystal violet staining was performed as described previously ${ }^{10}$.

\section{Clonogenicity assays}

For colony- and sphere-formation assays, $2500 \mathrm{cells} / \mathrm{ml}$ were seeded in TSM with or without $10 \%$ FCS, respectively, and treated with the indicated inhibitors. Gliomaspheres were stained as previously described ${ }^{6}$, scanned, and analyzed using the particle analyzer plugin from Image $^{11}$.

\section{Migration and invasion assays}

Migration and invasion assays had been performed and analyzed as previously described ${ }^{6}$. Briefly, 5000 cells of 48$\mathrm{h}$ pretreated cells in FGF-depleted TSM-work medium were used, and migration was analyzed after $24 \mathrm{~h}$.

\section{Quantitative real-time PCR (qPCR) and western blotting}

RNA was extracted using the ReliaPrep ${ }^{\mathrm{TM}}$ RNA Cell Miniprep System (Promega, Walldorf, Germany) according to the manufacturer's instructions. cDNA synthesis followed by qPCR with the PowerUp SYBR Green Mastermix (Thermo Fisher Scientific, Osterode am Harz, Germany) and western blotting was performed as previously described ${ }^{6,12}$. Oligonucleotides and antibodies can be found in the supplemental materials and methods.

\section{Chromatin immunoprecipitation followed by next- generation sequencing}

ChIP-seq was performed as previously described ${ }^{13}$. Briefly, cells were cross-linked in $1 \%$ formaldehyde followed by quenching with glycine (final concentration $125 \mathrm{mM}$ ), lysed, and sonicated for 30 cycles $(30 \mathrm{~s}$ on/30 s off) (Bioruptor pico, Diagenode, Liege, Belgium). Precleared chromatin was incubated with $1 \mu \mathrm{g}$ of antibody (for antibodies see supplemental materials and methods). Protein A-sepharose beads were used to pull down the antibody-chromatin complex, and samples were then decross-linked, and DNA was extracted.

\section{Library preparation and next-generation sequencing}

Libraries from RNA were synthesized using the TruSeq RNA Library Prep Kit v2 (Illumina, Munich, Germany) according to the manufacturer's instructions. Microplex Library preparation kit v2 (Diagenode) was used to prepare libraries from ChIP DNA. Pools of libraries were sequenced in the Transcriptome and Genome Analysis Laboratory (TAL) at the University Medical Center Göttingen using HiSeq4000 (Illumina, 50SE).

\section{Bioinformatic analysis}

Primary ChIP and RNA-seq analysis were performed as in ref. ${ }^{13}$. SEs were called using the ROSE algorithm ${ }^{8}$ by sorting enhancers based on the H3K27ac signal and using default settings with ignoring regions within $2.5 \mathrm{~kb}$ of annotated TSS. Associated genes were identified using GREAT analysis, and enriched factors at SE regions were identified using ReMAP ${ }^{14,15}$. Gene set enrichment analysis was performed using FPKM values of expressed genes in various conditions with SE selected as a text entrybased gene database. Clustering of gene expression patterns of SE-associated genes was performed using short time-series expression miner (STEM). 


\section{Results}

H3.3K27M-mutated DIPG cells display strong stem-like potential and proliferation activity compared with H3WTpedHGG cells

To evaluate if H3K27M mutation leads to changes in cell growth in vitro, we investigated the potential of $\mathrm{H} 3$ wild-type (H3WT) and H3K27M-mut pedHGG and DIPG cell lines to form gliomaspheres. In accordance with previous reports demonstrating that $\mathrm{H} 3 \mathrm{~K} 27 \mathrm{M}$ mutation confers a more stem cell-like phenotype ${ }^{2}$, we observed a stronger potential of the H3.3K27M-mutant VUMCDIPG-A and HSJD-DIPG-007 cell lines to form gliomaspheres under stem cell conditions. In contrast, spheroid formation of the primary DIPG cell line VUMC-DIPG-10 and the pedHGG cell lines SF188 and HSJD-GBM-001 harboring H3WT was less efficient (Fig. 1a, c). Consistently, we observed a strong expression of the stem cell markers Oct4, Sox2, and Nestin in H3.3K27M-containing DIPG cell lines. Conversely, expression was reduced or absent in H3WT-pedHGG and H3WT-DIPG gliomasphere cell lines (Fig. 1g). The expression of Oct4 and Sox2 was strongly reduced in all monolayer cells after culture under differentiation conditions for 3 days, but still slightly more expressed in H3.3K27M-mut-DIPG in comparison with H3WT-pedHGG cells (Supplemental Fig. 1).

Interestingly, H3.3K27M-mut-DIPG cells, grown either as a differentiating monolayer or in stem cell-like gliomaspheres, displayed a higher proliferation rate than H3WT-pedHGG/DIPG cells (Fig. 1d, f and Supplemental Fig. 2B). The higher proliferation potential of H3.3K27Mmut-DIPG was not accompanied by a stronger susceptibility to irradiation in comparison with primary H3WTpedHGG cells. On the contrary, sphere formation of H3.3K27M-mut-DIPG cells was almost unaffected by irradiation, indicating an additional mechanism of resistance in H3.3K27M-mutant DIPG, which is missing in H3WT-pedHGG/DIPG (Fig. 1b, c). These findings indicate the importance of H3K27M mutation for tumor phenotype and resistance to radiotherapy in H3.3K27Mmutated DIPG.

\section{Combinatorial inhibition of BET and CBP activity markedly affects proliferation of H3.3K27M-mut-DIPG cells}

Given the proposed importance of enhancer activation in DIPG, we next tested the effects of inhibition of CBP or BET proteins. Cell viability of H3.3K27M-mut-DIPG and H3WT-pedHGG gliomaspheres and monolayer cells was reduced upon $\mathrm{CBP}$ or BET protein inhibition in a dosedependent manner (Fig. 2a). Combined treatment with the CBP inhibitor ICG-001 and the BET inhibitor JQ1 led to the strongest effects in monolayer cells of H3.3K27Mmut-DIPG and H3WT-pedHGG gliomaspheres. Notably, additive effects of ICG-001 and JQ1 were observed in gliomaspheres, irrespective of the $\mathrm{H} 3$ mutation status (Fig. 2b, c). Comparison of cell quantification by crystal violet staining, proliferation by BrdU incorporation, and MTT analyses confirmed the reduced cell viability following BET and CBP inhibition (Supplemental Fig. 2A, B). Interestingly, JQ1 alone or in combination with ICG-001 induced apoptosis in H3WT-pedHGG cells as determined by PARP cleavage. Notably, in spite of their similar response to treatment, no apoptosis was observed in H3.3K27M-mut-DIPG cells.

\section{Combinatorial inhibition of CBP and BET proteins} attenuates tumor-associated characteristics of H3.3K27Mmut-DIPG

Given their marked effect on proliferation, we further investigated the function of ICG-001 and JQ1 in H3K27Mmut-DIPG cells. BRD4 was shown to be essential for maintaining stemness characteristics in gastric cancer and embryonic stem cells ${ }^{16,17}$. Due to high spheroid-forming potential in H3.3K27M-mut-DIPG, we tested if BET and CBP inhibition exerts inhibitory effects on these tumorassociated characteristics. Indeed, colony- and sphereformation assays demonstrated decreased self-renewal potential and proliferation upon treatment with ICG-001 and/or JQ1 (Fig. 3a, b). DIPG are also characterized by strong migration and invasion potential. Notably, inhibition of CBP decreased migration and invasion, while BET inhibition affected both to an even greater extent (Fig. 3c, d). Since radiotherapy is widely regarded as standard treatment for DIPG, we further tested whether BET and CBP inhibition may increase the radiosensitivity of DIPG cells (Fig. 3e-g). As expected, irradiation was most effective in differentiated monolayer DIPG cells in comparison with more slowly proliferating gliomaspheres (Fig. 3e, f and Fig. 1c, d). This phenotype is further associated with mechanisms that are protective against radiation-induced DNA damage ${ }^{18}$. Remarkably, combined therapy attenuated cell growth to an extent comparable to radiotherapy alone. Moreover, BET inhibition in DIPG gliomaspheres led to a stronger susceptibility to irradiation (Fig. 3f, g), which was supported by a strong reduction in self-renewal (Fig. 3b). These observations further demonstrate the positive effect of combinatorial inhibition of BET and CBP for DIPG treatment, especially after a prolonged incubation time of overall 5 days.

\section{Identification of super enhancer programs in DIPG cells}

SE have previously been implicated in tumor progression and aggressiveness in DIPG ${ }^{7}$. However, little is known about the SE landscape in H3.3K27M cells. Accordingly, we performed ChIP-seq for H3K27ac and H3K27me3 in DIPG-007 cells and identified SE regions (Fig. 4a). As expected, these regions were highly occupied by H3K27ac and were devoid of H3K27me3 (Fig. 4b). Comparison of 
A

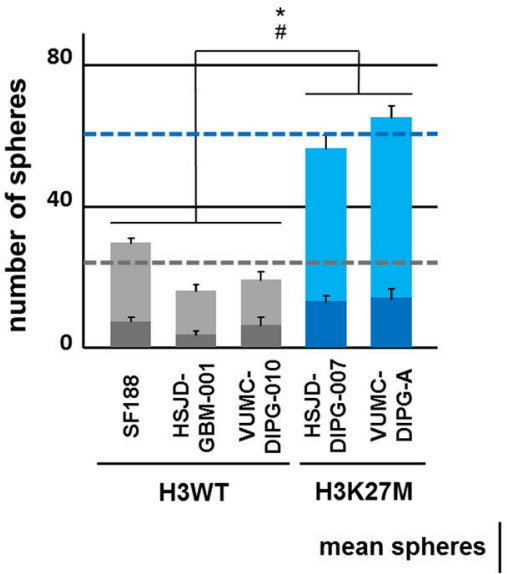

B

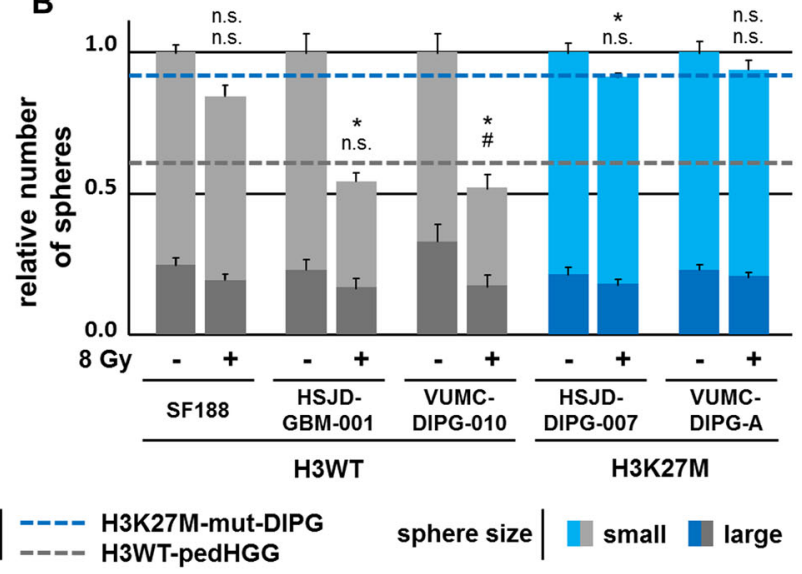

C

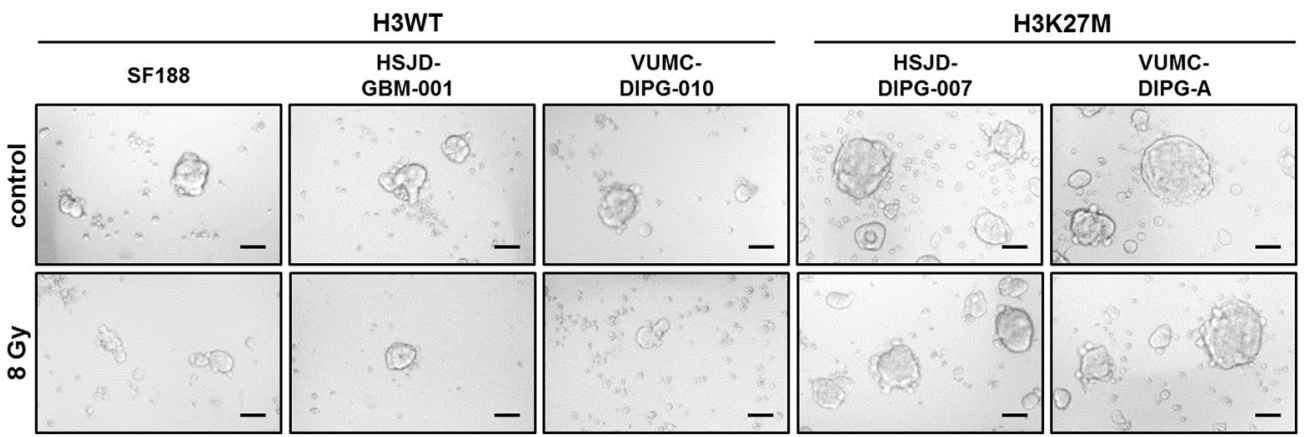

D

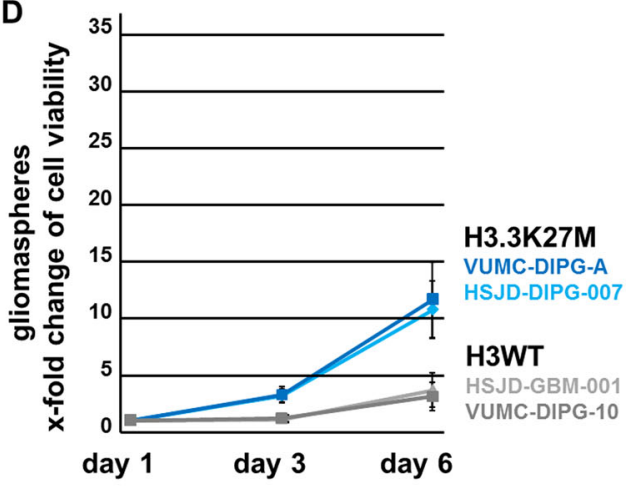

E

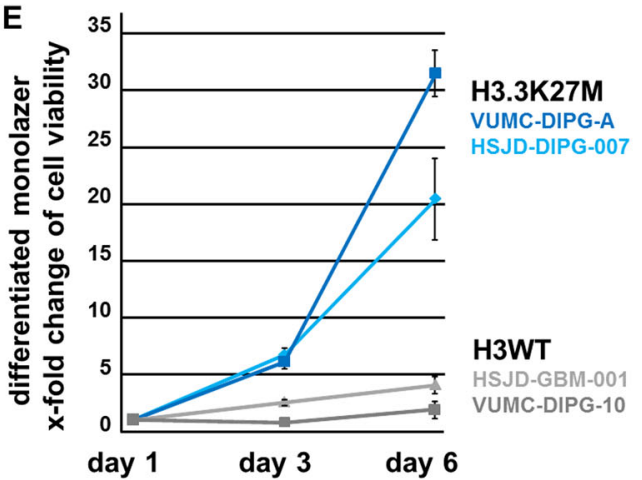

$\mathbf{F}$
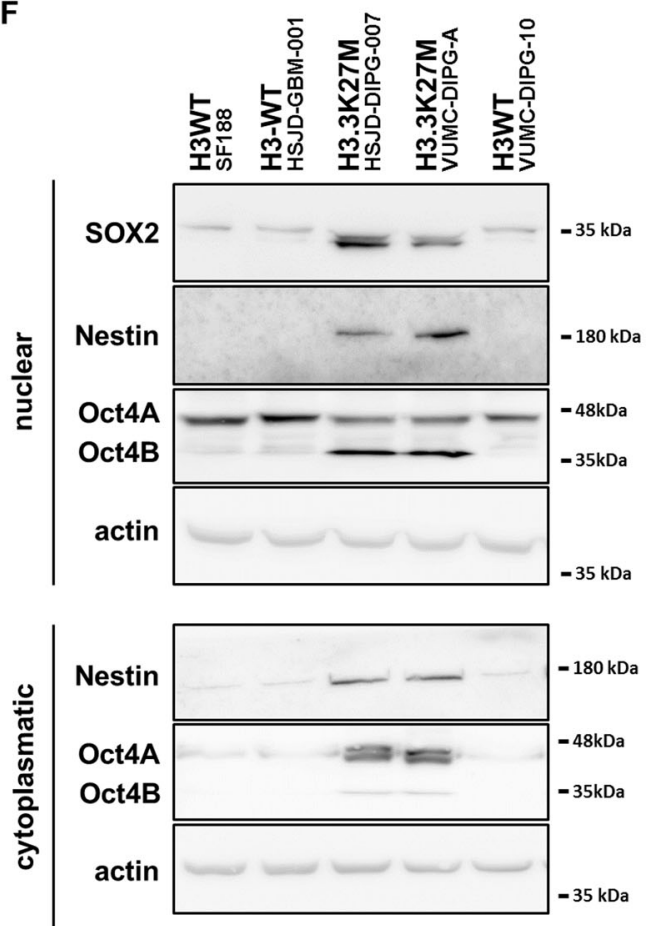

Fig. 1 (See legend on next page.) 
(see figure on previous page)

Fig. 1 H3.3K27M-mut-DIPG cells show higher proliferation, stem cell-like characteristics, and resistance to irradiation in comparison with H3WT-pedHGG cells. a Total number of H3WT and H3.3K27M-mut gliomaspheres after 5 days. b Quantification and (c) bright-field images of H3WT and H3.3K27M-mut gliomasphere formation after irradiation with $8 \mathrm{~Gy}$ (after $24 \mathrm{~h}$ ) after 5 days. Spheres where scored according to their size: large $(>0.1 \mathrm{~mm})$ and small $(<0.1 \mathrm{~mm})$, \# large and $\Delta$ small spheres, scale bar $1 \mathrm{~mm}$. d, e Cell viability assessed by MTT assay, reflecting the cell growth over time of primary H3.3K27M-mut-DIPG and H3WT-pedHGG/DIPG cells grown under stemness- (gliomaspheres) and differentiation- (monolayer) conditions. f Protein expression of stemness-associated markers Oct4, Nestin, and Sox2 in cytoplasmatic and nuclear protein fractions of H3WT and H3.3K27M gliomaspheres, assessed by western blotting, $\beta$-actin served as loading control.

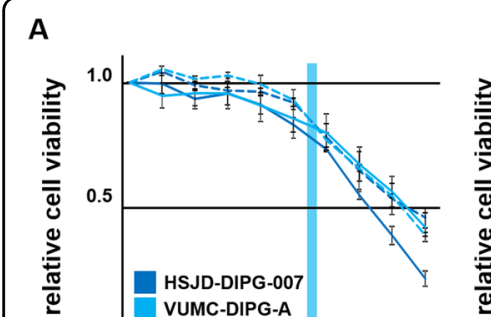

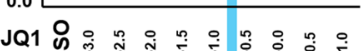

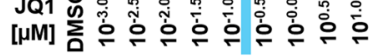
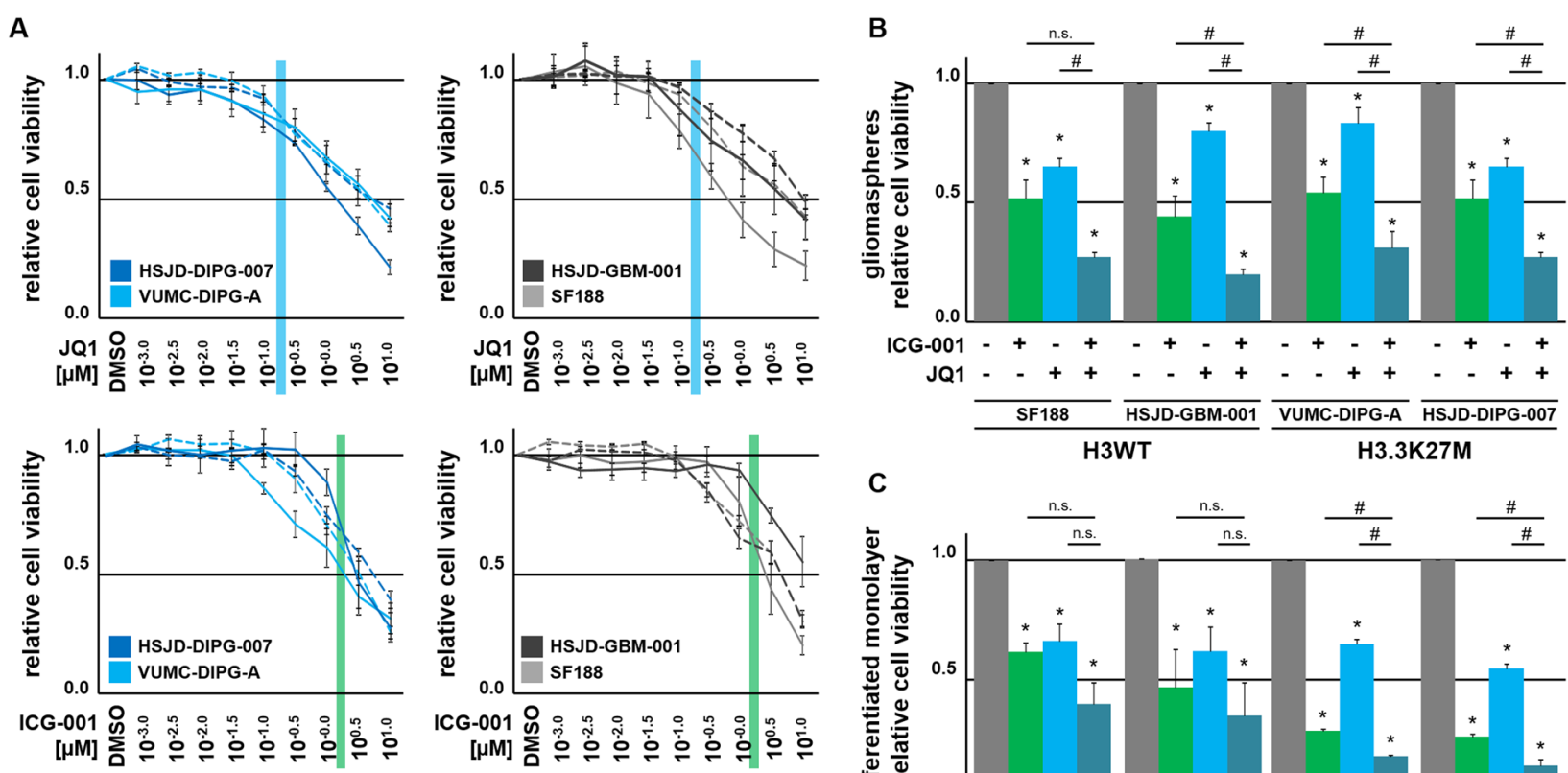

\begin{tabular}{c|c}
$\begin{array}{c}\text { concentration used for } \\
\text { further experiments }\end{array}$ & ICG-001 \\
JQ1
\end{tabular}

$$
\text { phenotype } \mid \begin{aligned}
& = \pm \text { gliomaspheres } \\
& =\text { monolayer }
\end{aligned}
$$

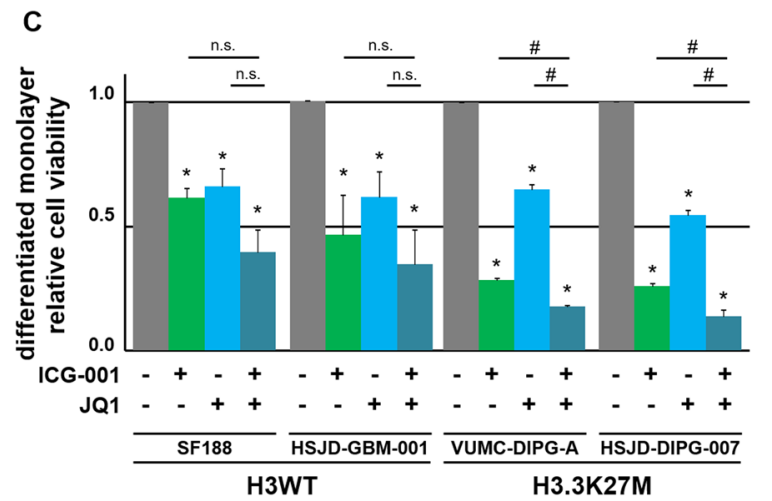

D

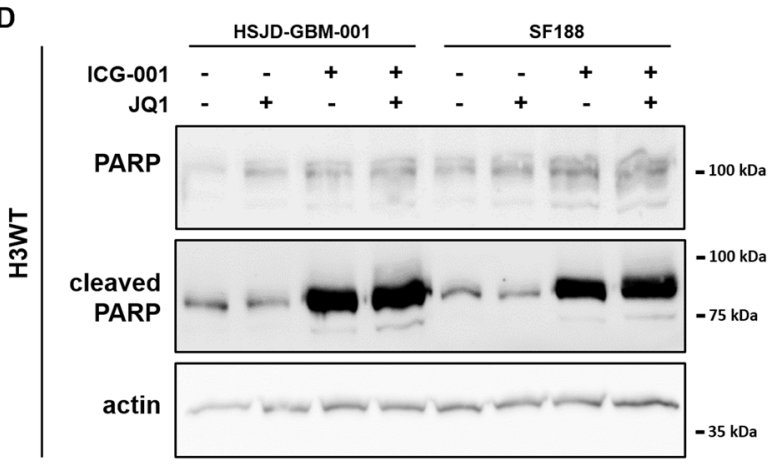

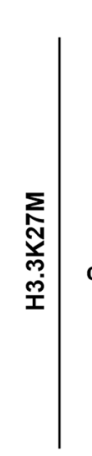

Fig. 2 Cell viability is strongly dependent on CBP and BET function. a MTT cell viability dilution curves with increasing concentrations of JQ1 and ICG-001, as indicated. H3K27M-mut-DIPG cells were treated with ICG-001 and JQ1, as indicated and subjected to MTT cell viability assay with H3K27M-mut-DIPG cells grown under (b) stemness- (gliomaspheres) and (c) differentiation- (monolayer) conditions. * $p<0.05$ with respect to DMSOtreated control cells, $\# p<0.05$ with respect to ICG-001 or JQ1-treated cells, as indicated. $\mathbf{d}$ Protein expression of cleaved PARP apoptosis marker in H3WT-pedHGG and H3K27M-mut-DIPG cells after ICG-001 and JQ1 treatment, as indicated; $\beta$-actin served as loading control.

the identified SE with published data from DIPG-007 cells with ectopic H3WT overexpression ${ }^{19}$ confirmed that the identified SE regions are significantly dependent upon
H3K27M in DIPG (Fig. 4c). Pathway enrichment analysis for genes associated with these SE revealed a c-MYC signature, which is in concordance with previous reports 


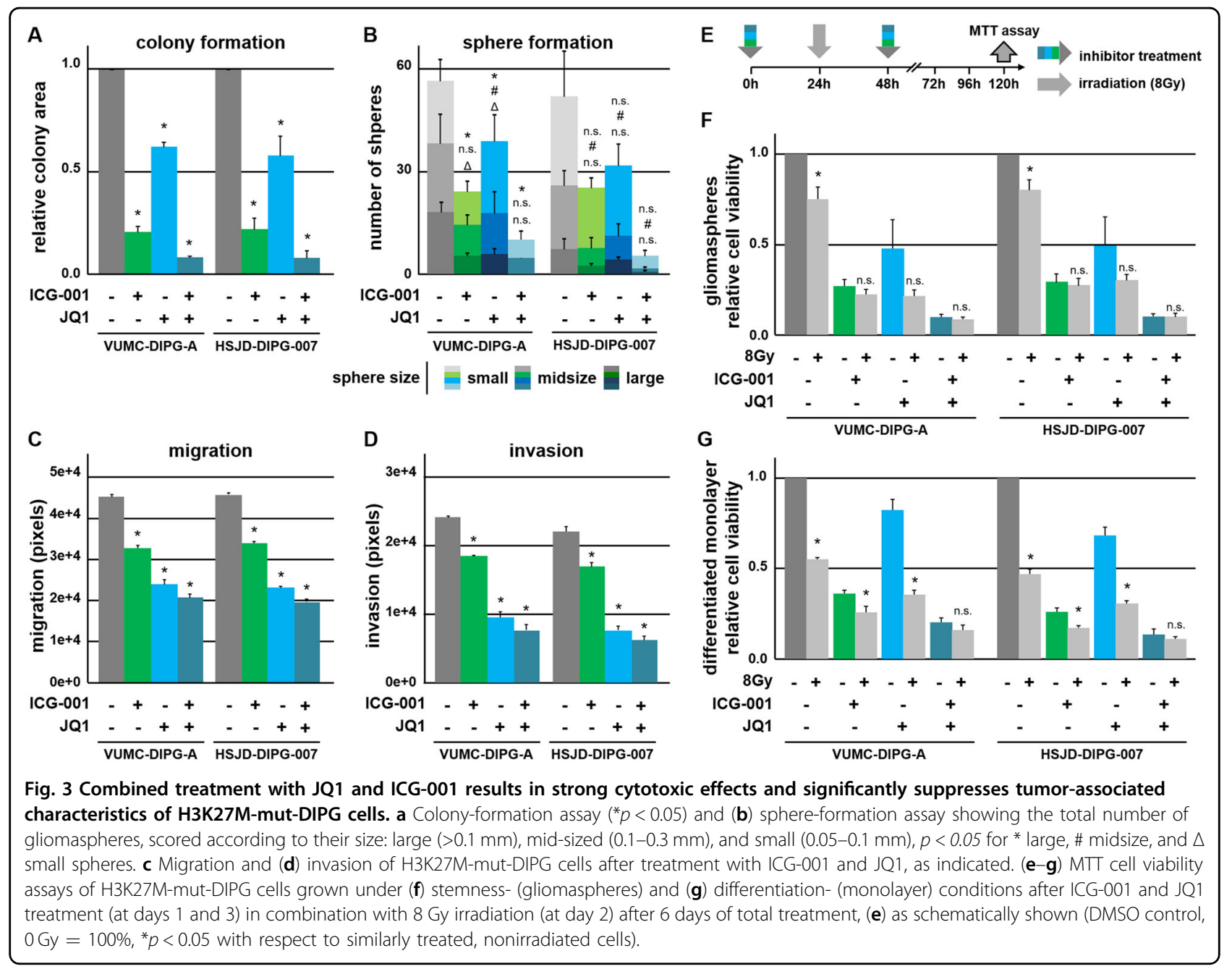

highlighting the role of MYC in DIPG (Fig. 4d) ${ }^{20,21}$. Additional enriched pathways included p73, which has previously been associated with a more invasive signature in glioblastomas ${ }^{22}$. To further characterize these SE, we used ReMAP to identify transcription factors that may potentially nucleate these regions (Fig. 4e). Interestingly, MYC and MYC-associated factor X (MAX) were identified among the most significantly enriched factors. Other transcription factors included the AP1 factor JUND, which has been found to mediate detrimental tumor biological effects in glioblastomas ${ }^{23}$. In addition, Transcription Factor 7 Like 2 (TCF7L2), which directly interacts with $\mathrm{CBP}^{24}$, was found to be highly enriched at these SE regions. In general, identification of SE in the DIPG-007 can uncover potential therapeutic targets and pathways that can be leveraged to increase survival in DIPG patients.

\section{Activation of a subset of SE by inhibition of CBP or BET can be reversed by combined therapy}

As BET inhibition was extensively documented to specifically downregulate SE programs in various cancer types including DIPG ${ }^{7,8}$, we hypothesized that the combinatorial effects we observed with BET and CBP inhibition may be due to potentiated effects in silencing the $\mathrm{SE}$ programs. Accordingly, we performed RNA- and ChIPseq analyses from cells treated with JQ1, ICG-001, or their combination. As expected, GSEA analysis showed that JQ1 treatment significantly downregulated the majority of genes associated with SE (Fig. 5a). In contrast, analysis in ICG-001-treated cells revealed that most genes associated with SE in DIPG-007 cells were significantly upregulated (Fig. 5b). Interestingly, combined therapy led to a lessweighted pattern of regulation with half of the genes observed to be upregulated compared with DMSO and the other half downregulated (Fig. 5c). To further characterize the altered patterns of regulation in combined therapy, we clustered the gene expression changes in SEassociated genes using the STEM algorithm and identified four significant clusters (Fig. 5d). Cluster 1 included 58 genes that were specifically downregulated by BET inhibition and unperturbed by CBP inhibition whether alone or in combination. These genes included many that are 

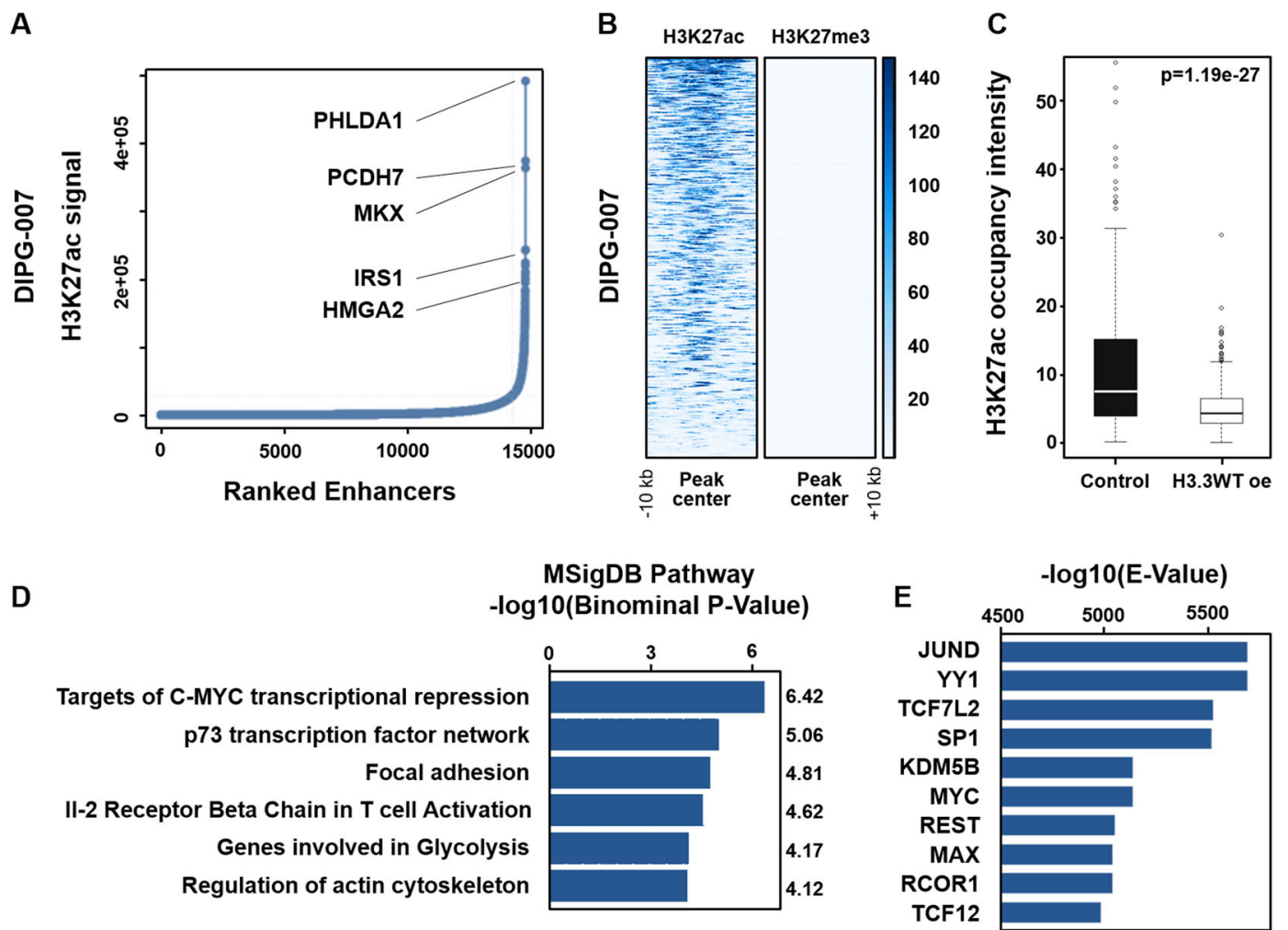

Fig. 4 Identification of super enhancer programs in H3.3K27M DIPG cells. a Output of the ROSE algorithm depicting ranking of enhancer regions based on H3K27ac after stitching with individual example genes shown. b Affinity profile plot for H3K27ac at super enhancer regions showing high occupancy of H3K27ac and no occupancy of H3K27me3. c Box plot of H3K27ac mean intensity at super enhancer regions ( $\pm 5 \mathrm{~kb}$ ) from publicly available data in control DIPG-007 cells and DIPG-007 with overexpression of wild-type H3.3. d Pathway enrichment performed by GREAT analysis on genes associated with super enhancers showing programs activated in DIPG-007. e ReMAP analysis output identifying the factors that most significantly nucleate super enhancer regions in DIPG-007.

known to be associated with a more aggressive and invasive phenotype in various cancer types including glioblastoma. Cluster 2 included 20 genes that are downregulated in all conditions. Importantly, cluster 3 included 23 genes that were upregulated by JQ1 and downregulated by ICG-001. Surprisingly, genes in this cluster were associated with an unfavorable prognosis in glioma, like Annexin A2 $(A N X A 2)^{25}$ and Ferritin Light Chain $(F T L)^{26}$. Interestingly, the fourth cluster comprised 11 genes whose upregulation by ICG-001 was prevented by concomitant treatment with JQ1. This cluster also included detrimental genes that are associated with poor survival and invasion, such as Aldehyde Dehydrogenase 1 family member A3 $(A L D H 1 A 3)^{27,28}$, Keratin 80 $(K R T 80)^{29}$, and Ras Responsive Element Binding Protein 1 $(R R E B 1)^{30}$ (for summary see Fig. 5e). qPCR analyses of H3K27M-DIPG-007 and H3WT-HSJD-GBM-001 cells confirmed that these genes are upregulated by CBP inhibition, but this activation is reversed by combinatorial treatment due to high sensitivity to BET inhibition (Fig. 6a-c). The same pattern of activation was observed for the super enhancers associated with these genes as confirmed by ChIP-seq following inhibition of BET and CBP monotherapy (Fig. 6d-f). These observations point to the activation of detrimental super enhancers by single treatment with either JQ1 or ICG-001, whereas combined treatment reverses these inadvertent programs (for summary see Fig. 6g).

\section{Discussion}

DIPG are the most aggressive brain tumors among pediatric high-grade glioma with median survival rates of less than 1 year. Due to their diffuse growth and location, DIPG are inoperable, and the current therapeutic gold standard remains to be radiation therapy (RT) that confers only a few months survival advantage. In 2012, identification of frequently occurring H3K27M mutations in up to $85 \%$ of all DIPG uncovered a shift of the epigenetic balance reflected by global loss of $\mathrm{H} 3 \mathrm{~K} 27 \mathrm{me} 3^{1}$. Interestingly, H3.3K27M in DIPG is not associated with an unfavorable prognosis compared with DIPG carrying H3WT, indicating that additional factors may contribute to the poor prognosis in DIPG ${ }^{1}$. In contrast, survival differences are observed between H3K27M mutant and 
A DIPG Super Enhancers

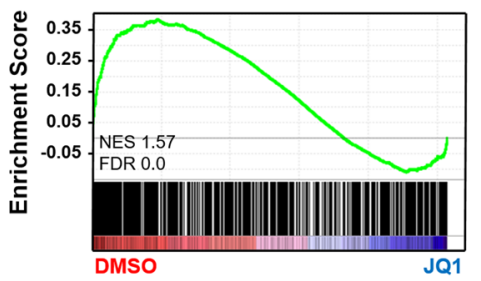

B

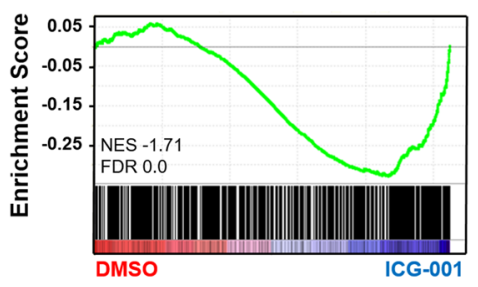

C

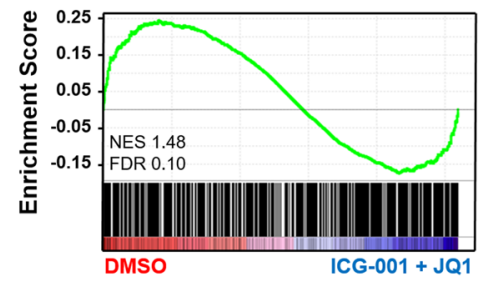

D

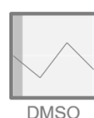

Cluster 1
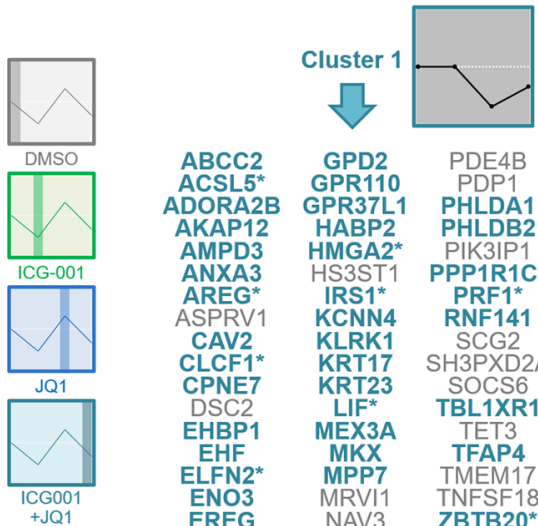

GPR110

ACSL5

ADORA2B GPR37L1

$\begin{array}{lll}\text { ADORA2B } & \text { GPR37L1 } & \text { PHLDA1 } \\ \text { AKAP12 } & \text { HABP2 } & \text { PHLDB2 } \\ \text { AMPD3 } & \text { HMGA2* } & \text { PIK3IP1 }\end{array}$

PIK3IP1

IRS1* PPRT1*

ASPRV1 KCNN4 RNF141

CAV2 KLRK1 SCG2

CLCF1* KRT17 SH3PXD2A

CPNE7

DSC2

EHBP1

EHF

ELFN2*

EREG

ERRFI1

FBLIM1

KRT23

MEX3A

MEX3A
MKX

MPP7

MRVI1
NAV3

NAV3
NR2F1

$\mathrm{PCDH} 7$

SOCS6

TBL1XR

TET3
TFAP4

TMEM17

TNFSF18

ZBTB20*

ZMYND8

ZNF462

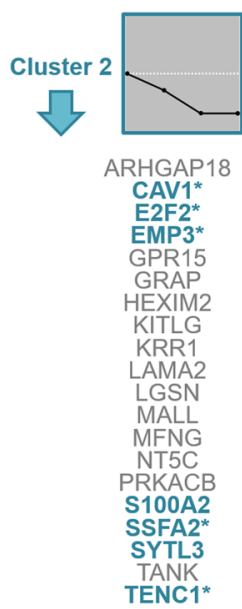

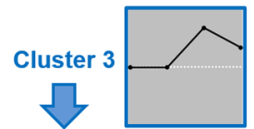

ABCA7

ACAP 3

ANXA2*

CCDC85

EPPK1

FTL*

HIST1 1 2BC

HMGA1*

ID1*

ID3*
IFNE

ISYNA1

KRT8

MIDN

PLEKHM

PNKP

RNF223

TUBB3*

ZDHHC22

ZFP36
E

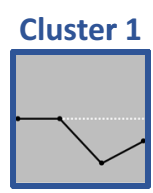

ACSL5

AREG

CLCF1

ELFN2

EREG

FBLIM1

HMGA2

IRS1

LIF

PPP1R1C

PRF1

ZBTB20

Cluster 2

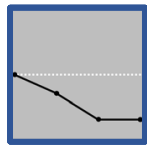

CAV1

E2F2

EMP3

SSFA2

TENC1

Cluster 3

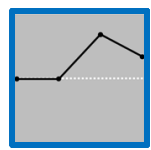

ANXA2

HMGA1

ID1

ID3

TUBB3

FTL

Cluster 4

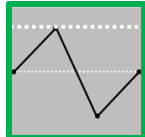

ALDH1A3

FGF1

MTHFD1L

NFATC2 induces glioma cell proliferation and tumorigenicity [53]

associated with patient survival and astrocytoma's malignancy [54]

associated with poor patient survival [55]

oncogenic function [56]

enhancess tumorigenicity by activation of ERK/MAPK [57]

promotes migration and invasion by activation of STAT3 [58]

promotes stemness, invasion, poor survival and tumorigenicity [59]

promotes radioresistence [60]

contributes to tumor growth and progression [41]

involved in disease progression and resistance to TMZ therapy [61]

associated with poor patient survival [62]

promotes proliferation, migration and invasion [63]

promotes tumorigenicity [64]

regulates the tumor-initiating capability (proliferation) [65]

associated with GBM patient survival [42]

promotes proliferation of GBM [66]

contributes to the maintenance of GBM structures [67]

positive regulator of the mesenchymal subtype [26]

enhances stemness and temozolomide resistance [68]

regulates GBM self-renewal and radio-resistance [69]

promotes formation of stem-like cells and tumor angiogenesis [70]

induces tumor progression and malignant transformation [71]

induces proliferation [27]

induces mesenchymal identity, predictive for survival of patients [25]

sustains stem-cell characteristics [72]

promotes invasion [73]

promotes invasion [74]

Fig. 5 CBP and BET inhibition downregulates super enhancer programs inadvertently activated by individual therapies. a-c GSEA plots showing enrichment of super enhancer programs in control compared with treatment (a) of JQ1, (b) ICG-001, and (c) combinatorial treatment. d Significant clusters showing different patterns of gene expression regulation upon treatment with DMSO, JQ1, ICG-001, or combined ICG-001/JQ1 treatment. Asterisks mark genes that promote GBM characteristics. Bold and colored genes promote other tumor entities. e Genes of four clusters differentially regulated by ICG-001, JQ1, and combinatorial treatment and their function in GBM pathogenesis ${ }^{52-73}$. 


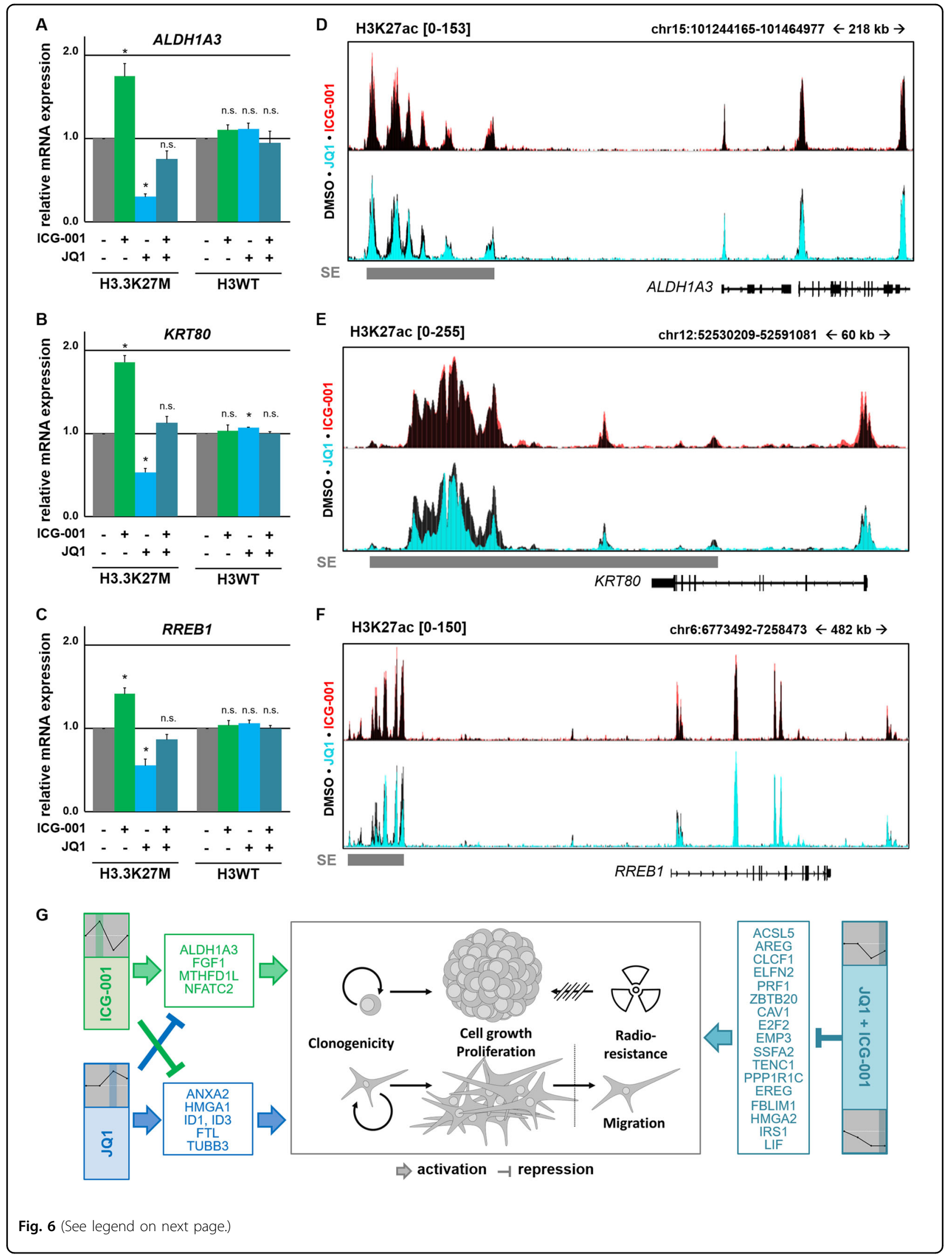


(see figure on previous page)

Fig. 6 Inadvertently activated gene expression in DIPG-007 cells by single treatment with ICG-001 and JQ1 is reversed by combined treatment due to inhibition of associated super enhancers. a-c qPCR validating the gene expression of (a) ALDH1A3, (b) KRT80, and (c) RREB1 in DIPG-007 and GBM-001 cells after $48 \mathrm{~h}$ of treatment with ICG-001 and JQ1, as indicated. $P<0.05$. d-f Occupancy profiles of H3K27ac at ALDH1A3, KRT80, and RREB1 in cells treated with JQ1 or ICG-001 overlaid on DMSO-treated cells for comparison. Super enhancers in control cells are shown in gray. $\mathbf{g}$ Graphical model depicting the mutual effect of JQ1 and ICG-001 on detrimental programs affecting each other and leading to an increased effect of combinatorial therapy.

H3 wild type in non-pontine diffuse midline gliomas ${ }^{31}$. This suggests that H3.3K27M mutation might play a more prominent role in non-pontine midline gliomas compared with DIPG. However, H3K27M-mutated DIPG cell lines appear to behave in general more aggressively in vitro compared with H3WT-DIPG and pedHGG cells confirming that additional factors contribute to the poor survival of DIPG patients. H3K27me3-deficient nucleosomes in H3K27M-mut-DIPG acquire H3K27ac, resulting in the formation of H3K27M-K27ac heterotypic nucleosomes and global hyperacetylation of H3K27.

Hyperacetylation favors the function of transcriptionally active, acetylation-dependent factors such as BET proteins ${ }^{5}$. Previous investigations showed that BET inhibition results in DIPG growth suppression in vitro and in vivo ${ }^{5,7,32,33}$. Consistently, we showed that H3.3K27Mmut-DIPG cells are highly sensitive to JQ1. Moreover, gene expression profiling in H3.3K27M-mut-DIPG cells revealed that many oncogenic programs are attenuated by JQ1 treatment. On the other hand, we hypothesized that acetyltransferases such as CBP may play a significant role in the context of DIPG malignancy as it was reported to highly occupy super enhancers ${ }^{34}$. Indeed, H3WTpedHGG as well as H3.3K27M-mut-DIPG cell growth was sensitive to $\mathrm{CBP}$ inhibition. Interestingly, monolayer cultures of H3.3K27M-mut-DIPG cells grown under differentiation conditions showed a stronger response to treatment with ICG-001. Notably, CBP was shown to be crucial for the activation of genes that promote normal differentiation of cortical precursors into neuronal, astrocytic, and oligodendroglial precursors ${ }^{35}$. However, the particular function of CBP in this context in DIPG needs to be further examined.

Moreover, we further investigated the combined effect of CBP and BET inhibition on H3.3K27M-mut-DIPG cells. Surprisingly, we found that the combinatorial effect of JQ1 and ICG-001 efficiently hinders proliferation, sphere formation, and radioresistance with strongest effects after combinatory treatment. This was not expected given the relatively moderate effects after monotherapy. Determination of the expression changes and affected SE by single and combinatorial use of BET and CBP inhibitors revealed partially antagonistic and detrimental effects caused by ICG-001 or JQ1, which were eliminated after combination of these inhibitors. As expected, JQ1 treatment significantly downregulated most genes associated with $\mathrm{SE}$, which we identified in H3.3K27M-mut-DIPG cells. In contrast, we observed that monotherapy with ICG-001 upregulates the majority of $\mathrm{SE}$-associated genes. This effect is quite surprising, given the reported enrichment of $\mathrm{CBP}$ as a feature for $\mathrm{SE}^{36}$. However, ICG-001 does not inhibit CBP catalytic activity, but instead blocks interaction with other proteins such as $\beta$-catenin and $\operatorname{RXR}^{37}$. Consequently, it is possible that inhibition of such interactions may facilitate others, thereby promoting the nucleation of SE driven by factors whose interaction with CBP is not inhibited by ICG-001. Thus, it would be of interest to evaluate the effects of newly developed CBP inhibitors that exhibit high selectivity and target the catalytic region of $\mathrm{CBP}^{38}$. In addition, we uncovered that JQ1 and ICG-001 upregulate genes that are associated with a more aggressive phenotype. Importantly, this effect is attenuated when these agents are used in combination.

A similar effect, where the expression of genes upregulated by one agent is blocked by BET inhibition, has already been reported for JQ1 treatment in conjunction with histone deacetylase inhibitors ${ }^{7,39}$. In general, this provides a rational basis for further investigation of combined ICG-001/BET inhibitor treatment as a potential effective therapeutic approach in DIPG.

Interestingly, we identified four different, significant gene sets by clustering the observed gene expression changes in SE-associated genes after single and combined inhibition of CBP and BET proteins. Cluster 1 included downregulated genes by JQ1 and in combination with ICG-001. Cluster 2 included downregulated genes in all conditions. Genes identified in these two clusters are associated with a more aggressive and invasive phenotype in various cancer types including glioblastoma. For example, Leukemia inhibitory factor (LIF) in Cluster 1 that contributes to GBM tumor growth and progression ${ }^{40}$ or Epithelial membrane protein 3 (EMP3) in Cluster 2 whose high expression was shown to be associated with worse GBM patient survival ${ }^{41}$. Genes of Cluster 3 were upregulated by JQ1 and downregulated by ICG-001, and Cluster 4 included genes whose upregulation by ICG-001 was prevented by concomitant treatment with JQ1. Surprisingly, genes in clusters 3 and 4 were associated with an unfavorable prognosis in glioma. For example, Annexin 
A2 (ANXA2), Ferritin Light Chain (FTL), and Inhibitor of DNA-binding-1 (ID1) fulfill important tumor functions in glioma and glioblastoma, such as the promotion of invasion and tumor progression ${ }^{42}$, proliferation ${ }^{26}$, or chemoresistance ${ }^{43-45}$. The fourth cluster included detrimental genes such as $A L D H 1 A 3$ promoting stemness-like features in glioma, and is associated with worse survival ${ }^{27,28}$. Another example, KRT80, is overexpressed in more aggressive glioma ${ }^{46}$ and, together with CBP, contributes to therapy resistance ${ }^{47}$, whereas RREB1 promotes an invasive phenotype $e^{30,48}$. In agreement with the function of these genes, ICG-001 treatment of H3.3K27M-mut-DIPG was not as efficient in inhibiting migration and invasion as JQ1 treatment. Interestingly, gene expression profiling upon BET and CBP inhibition revealed that this effect appears to be particularly specific to H3K27M-mut-DIPG cells.

Accordingly, we propose a model where JQ1 and ICG001 not only inactivate SE-related programs when given alone, but also inadvertently activate detrimental programs that can be attenuated when both agents are used in combination. However, further investigations are needed to elucidate if JQ1, ICG-001, or similar drugs with the same function can pass the blood-brain barrier (BBB) and inhibit DIPG growth in vivo. Recent studies have already successfully proved the BBB permeability of JQ1 $1^{49,50}$. Although ICG-001 has not been used to treat brain malignancies so far, its derivative PRI-724 has already been investigated in several clinical trials in other types of cancer, including myeloid malignancies, pancreatic cancer, and advanced solid tumors (NCT01302405, NCT01606579, and NCT01764477). Notably, PRI-724 leads to comparable effects to those seen with ICG-001 in DIPG and pedHGG cells (Supplemental Fig. 3A, B). ICG001 had been additionally found to transiently increase $\mathrm{BBB}$ permeability due to depression of endothelial cell-cell interaction ${ }^{51}$.

In conclusion, the combinatorial use of JQ1 and ICG001 showed a high efficacy in attenuating the growth and self-renewing potential of DIPG cells. In addition, JQ1 and ICG-001 exhibited an antiproliferative effect that is comparable to radiation alone. We report that ICG-001 and JQ1 inadvertently activate a subgroup of detrimental super enhancer programs that are reversed in combination.

\footnotetext{
Acknowledgements

The authors thank A. Herbst for technical and the DFG [grant number: 396708675], the University Medical Center, and Menschen für Kinder e.V. for financial support. We would like to thank G. Salinas, F. Ludewig, and S. Lutz (Transcriptome and Genome Analysis Laboratory, University Medical Center Göttingen) for performing the next-generation sequencing. Research was funded by the Deutsche Forschungsgemeinschaft (DFG) and Menschen für Kinder e.V. This work was supported by the Deutsche Forschungsgemeinschaft (DFG), Bonn, Germany [grant number: 396708675], by Menschen fuer Kinder e. V., Albshausen, Germany [no grant number available], and the Intramural
}

Research Funding Program of the University Medical Center Göttingen Germany [no grant number available].

\section{Author details}

'Division of Pediatric Hematology and Oncology, Department of Child and Adolescent Health, University Medical Center Goettingen, Robert Koch Straße 40, Goettingen, Germany. ${ }^{2}$ Gene Regulatory Mechanisms and Molecular Epigenetics Lab, Division of Gastroenterology and Hepatology, Mayo Clinic, 200 First Street SW, Rochester MN 55905, USA. ${ }^{3}$ Institute of Neuropathology, University Hospital Bonn, Venusberg-Campus 1, Bonn, Germany. ${ }^{4}$ Division of Pediatric Hematology/Oncology, Department of Pediatrics and Adolescent Medicine, Medical University of Graz, Auenbruggerplatz 38, Graz, Austria.

${ }^{5}$ Pediatric Hematology and Oncology, Hospital Sant Joan de Deu/Institut de Recerca, Sant Joan de Deu, Barcelona, Spain. ${ }^{6}$ Departments of Pediatric Oncology/Hematology, Cancer Center Amsterdam, VU University Medical Centers, Amsterdam, The Netherlands. ${ }^{7}$ Princess Máxima Center for Pediatric Oncology, Heidelberglaan 25, Utrecht, The Netherlands. ${ }^{8}$ Present address: Department of Radiation Oncology, Radiooncology/Radiobiology, Klinikum rechts der Isar, Technische Universität München, Ismaninger Str. 22, Munich, Germany

\section{Funding}

Open access funding provided by Projekt DEAL.

\section{Data availability}

Raw datasets for ChIP/RNA-seq were deposited in ArrayExpress (https://www. ebi.ac.uk/arrayexpress/) under the accession numbers E-MTAB-8242 for ChIPseq, E-MTAB-9152 and E-MTAB-8243 for RNA-seq.

\section{Conflict of interest}

The authors declare that they have no conflict of interest.

\section{Publisher's note}

Springer Nature remains neutral with regard to jurisdictional claims in published maps and institutional affiliations.

Supplementary Information accompanies this paper at (https://doi.org/ 10.1038/s41419-020-02800-7).

Received: 2 December 2019 Revised: 15 July 2020 Accepted: 15 July 2020 Published online: 21 August 2020

\section{References}

1. Hoffman, L. M. et al. Clinical, radiologic, pathologic, and molecular characteristics of long-term survivors of diffuse intrinsic pontine glioma (DIPG): a collaborative report from the International and European Society for Pediatric Oncology DIPG Registries. J. Clin. Oncol. 36, 1963-1972 (2018).

2. Silveira, A. B. et al. H3.3 K27M depletion increases differentiation and extends latency of diffuse intrinsic pontine glioma growth in vivo. Acta Neuropathologica 137, 637-655 (2019).

3. Lavarone, E., Barbieri, C. M. \& Pasini, D. Dissecting the role of H3K27 acetylation and methylation in PRC2 mediated control of cellular identity. Nat. Commun. 10, 1679 (2019).

4. Chan, H. M. \& La Thangue, N. B. p300/CBP proteins: HATs for transcriptional bridges and scaffolds. J. Cell Sci. 114, 2363-2373 (2001).

5. Piunti, A. et al. Therapeutic targeting of polycomb and BET bromodomain proteins in diffuse intrinsic pontine gliomas. Nat. Med. 23, 493-500 (2017).

6. Wiese, M. et al. The $\beta$-catenin/CBP-antagonist ICG-001 inhibits pediatric glioma tumorigenicity in a Wnt-independent manner. Oncotarget 8, 27300-27313 (2017).

7. Nagaraja, S. et al. Transcriptional dependencies in diffuse intrinsic pontine glioma. Cancer Cell 31, 635-652 e6 (2017).

8. Lovén, J. et al. Selective inhibition of tumor oncogenes by disruption of superenhancers. Cell 153, 320-334 (2013).

9. Meel, M. H. et al. Culture methods of diffuse intrinsic pontine glioma cells determine response to targeted therapies. Exp. Cell Res. 360, 397-403 (2017). 
10. Feoktistova, M., Geserick, P. \& Leverkus, M. Crystal violet assay for determining viability of cultured cells. Cold Spring Harb. Protoc. 2016, pdb.prot087379 (2016).

11. Rueden, C. T. et al. ImageJ2: ImageJ for the next generation of scientific image data. BMC Bioinforma. 18, 529 (2017).

12. Beg, A. A., Finco, T. S., Nantermet, P. V. \& Baldwin, A. S. Tumor necrosis factor and interleukin-1 lead to phosphorylation and loss of I kappa B alpha: a mechanism for NF-kappa B activation. Mol. Cell. Biol. 13, 3301-3310 (1993).

13. Hamdan, F. H. \& Johnsen, S. A. DeltaNp63-dependent super enhancers define molecular identity in pancreatic cancer by an interconnected transcription factor network. Proc. Natl Acad. Sci. USA 115, E12343-E12352 (2018).

14. McLean, C. Y. et al. GREAT improves functional interpretation of cis-regulatory regions. Nat. Biotechnol. 28, 495-501 (2010).

15. Chèneby, J., Gheorghe, M., Artufel, M., Mathelier, A. \& Ballester, B. ReMap 2018 : an updated atlas of regulatory regions from an integrative analysis of DNAbinding ChIP-seq experiments. Nucleic Acids Res. 46, D267-D275 (2018).

16. Song, $\mathrm{H}$. et al. BRD4 promotes the stemness of gastric cancer cells via attenuating miR-216a-3p-mediated inhibition of Wnt/ $\beta$-catenin signaling. Eur. J. Pharmacol. 852, 189-197 (2019).

17. Horne, G. A. et al. Nanog requires BRD4 to maintain murine embryonic stem cell pluripotency and is suppressed by bromodomain inhibitor JQ1 together with Lefty1. Stem Cells Dev. 24, 879-891 (2015).

18. Skvortsova, I., Debbage, P., Kumar, V. \& Skvortsov, S. Radiation resistance: cancer stem cells (CSCs) and their enigmatic pro-survival signaling. Semin. Cancer Biol. 35, 39-44 (2015).

19. Dahl, N. A. et al. Super elongation complex as a targetable dependency in diffuse midline glioma. Cell Rep. 31, 107485 (2020).

20. Khuong-Quang, D.-A. et al. K27M mutation in histone H3.3 defines clinically and biologically distinct subgroups of pediatric diffuse intrinsic pontine gliomas. Acta Neuropathologica 124, 439-447 (2012).

21. Sun, $Y$. et al. Potent anti-tumor efficacy of palbociclib in treatment-naïve H3.3K27M-mutant diffuse intrinsic pontine glioma. EBioMedicine 43, 171-179 (2019).

22. Landré, V., Antonov, A., Knight, R. \& Melino, G. p73 promotes glioblastoma cell invasion by directly activating POSTN (periostin) expression. Oncotarget 7 11785-11802 (2016)

23. Rong, $Y$. et al. Epidermal growth factor receptor and PTEN modulate tissue factor expression in glioblastoma through JunD/activator protein-1 transcriptional activity. Cancer Res. 69, 2540-2549 (2009).

24. Jin, T. Current understanding on role of the wnt signaling pathway effector TCF7L2 in glucose homeostasis. Endocr. Rev. 37, 254-277 (2016).

25. Kling, $T$. et al. Integrative modeling reveals annexin A2-mediated epigenetic control of mesenchymal glioblastoma. EBioMedicine 12, 72-85 (2016).

26. $\mathrm{Wu}$, T. et al. Expression of ferritin light chain (FTL) is elevated in glioblastoma, and FTL silencing inhibits glioblastoma cell proliferation via the GADD45/JNK pathway. PLoS ONE 11, e0149361 (2016).

27. Cheng, $P$. et al. FOXD1-ALDH1A3 signaling is a determinant for the selfrenewal and tumorigenicity of mesenchymal glioma stem cells. Cancer Res. 76, 7219-7230 (2016).

28. Li, G. et al. ALDH1A3 induces mesenchymal differentiation and serves as a predictor for survival in glioblastoma. Cell Death Dis. 9, 1190 (2018),

29. Chandrashekar, D. S. et al. UALCAN: a portal for facilitating tumor subgroup gene expression and survival analyses. Neoplasia (N. Y., N. Y.) 19, 649-658 (2017).

30. Koo, S., Martin, G. \& Toussaint, L. G. MicroRNA-145 promotes the phenotype of human glioblastoma cells selected for invasion. Anticancer Res. 35, 3209-3215 (2015).

31. Karremann, M. et al. Diffuse high-grade gliomas with $\mathrm{H} 3 \mathrm{~K} 27 \mathrm{M}$ mutations carry a dismal prognosis independent of tumor location. Neuro-Oncol. 20, 123-131 (2018).

32. Zhang, $Y$. et al. Combination of EZH2 inhibitor and BET inhibitor for treatment of diffuse intrinsic pontine glioma. Cell Bioscience 7, 56 (2017).

33. Taylor, I. C. et al. Disrupting NOTCH slows diffuse intrinsic pontine glioma growth, enhances radiation sensitivity, and shows combinatorial efficacy with bromodomain inhibition. J. Neuropathol. Exp. Neurol. 74, 778-790 (2015).

34. Suzuki, H. I., Young, R. A. \& Sharp, P. A. Super-enhancer-mediated RNA processing revealed by integrative MicroRNA network analysis. Cell $\mathbf{1 6 8}$ 1000-1014.e15 (2017)

35. Wang, J. et al. CBP histone acetyltransferase activity regulates embryonic neural differentiation in the normal and Rubinstein-Taybi syndrome brain. Dev. Cell 18, 114-125 (2010).
36. Hnisz, D. et al. Super-enhancers in the control of cell identity and disease. Cell 155, 934-947 (2013).

37. Emami, K. H. et al. A small molecule inhibitor of beta-catenin/CREB-binding protein transcription corrected. Proc. Natl Acad. Sci. USA 101, 12682-12687 (2004).

38. Lai, K. W. et al. Design and synthesis of a biaryl series as inhibitors for the bromodomains of CBP/P300. Bioorg. Medicinal Chem. Lett. 28, 15-23 (2018).

39. Carew, J. S. et al. Rational cotargeting of HDAC6 and BET proteins yields synergistic antimyeloma activity. Blood Adv. 3, 1318-1329 (2019).

40. Pascual-Garcia, M. et al. LIF regulates CXCL9 in tumor-associated macrophages and prevents CD8(+) T cell tumor-infiltration impairing anti-PD1 therapy. Nat Commun. 10, 2416 (2019).

41. Yue, H., Xu, Q. \& Xie, S. High EMP3 expression might independently predict poor overall survival in glioblastoma and its expression is related to DNA methylation. Medicine 97, e9538 (2018).

42. Zhai, $H$. et al. Annexin A2 promotes glioma cell invasion and tumor progression. J. Neurosci. 31, 14346-14360 (2011).

43. Soroceanu, L. et al. Id-1 is a key transcriptional regulator of glioblastoma aggressiveness and a novel therapeutic target. Cancer Res. 73, 1559-1569 (2013).

44. Sachdeva, R. et al. ID1 is critical for tumorigenesis and regulates chemoresistance in glioblastoma. Cancer Res. 79, 4057-4071 (2019).

45. Chen, D., Forootan, S. S., Gosney, J. R., Forootan, F. S. \& Ke, Y. Increased expression of Id 1 and Id3 promotes tumorigenicity by enhancing angiogenesis and suppressing apoptosis in small cell lung cancer. Genes Cancer $\mathbf{5}$ 212-225 (2014)

46. Zhang, G. et al. Clinical outcome of gliosarcoma compared with glioblastoma multiforme: a clinical study in Chinese patients. J. Neuro-Oncol. 127, 355-362 (2016).

47. Saldanha da Gama Fischer, Jde et al. Chemo-resistant protein expression pattern of glioblastoma cells (A172) to perillyl alcohol. J. Proteome Res. 10 153-160 (2011).

48. Kent, O. A. et al. Repression of the miR-143/145 cluster by oncogenic Ras initiates a tumor-promoting feed-forward pathway. Genes Dev. 24, 2754-2759 (2010).

49. Korb, E., Herre, M., Zucker-Scharff, I., Darnell, R. B. \& Allis, C. D. BET protein Brd4 activates transcription in neurons and BET inhibitor Jq1 blocks memory in mice. Nat. Neurosci. 18, 1464-1473 (2015).

50. Jung, $K$. H. et al. RNA sequencing reveals distinct mechanisms underlying BET inhibitor JQ1-mediated modulation of the LPS-induced activation of BV-2 microglial cells. J. Neuroinflammation 12, 36 (2015)

51. Vezina, A. \& Jackson, S. SCIDOT-21. Improving drug delivery to glioblastoma by targeting canonical wnt/B-catenin signaling in the blood-brain barrier. NeuroOncol. 21, vi275-vi276 (2019).

52. Pei, Z. et al. Acyl-CoA synthetase VL3 knockdown inhibits human glioma cell proliferation and tumorigenicity. Cancer Res. 69, 9175-9182 (2009).

53. Steponaitis, G. et al. Significance of amphiregulin (AREG) for the outcome of low and high grade astrocytoma patients. J. Cancer 10, 1479-1488 (2019).

54. Tejero, R. et al. Gene signatures of quiescent glioblastoma cells reveal mesenchymal shift and interactions with niche microenvironment. EBioMedicine 42, 252-269 (2019).

55. Liu, C. et al. LINC00470 coordinates the epigenetic regulation of ELFN2 to distract GBM cell autophagy. Mol. Ther.: J. Am. Soc. Gene Ther. 26, 2267-2281 (2018).

56. Kohsaka, S. et al. Epiregulin enhances tumorigenicity by activating the ERK MAPK pathway in glioblastoma. Neuro-Oncol. 16, 960-970 (2014).

57. Ou, Y. et al. Migfilin protein promotes migration and invasion in human glioma through epidermal growth factor receptor-mediated phospholipase Cgamma and STAT3 protein signaling pathways. J. Biol. Chem. 287, 32394-32405 (2012).

58. Kaur, H. et al. The transcriptional modulator HMGA2 promotes stemness and tumorigenicity in glioblastoma. Cancer Lett. 377, 55-64 (2016).

59. Li, D., Chi, G., Chen, Z. \& Jin, X. MicroRNA-1225-5p behaves as a tumor suppressor in human glioblastoma via targeting of IRS1. OncoTargets Ther. 11, 6339-6350 (2018).

60. Liu, L. et al. MicroRNA-182 targets protein phosphatase 1 regulatory inhibitor subunit 1C in glioblastoma. Oncotarget 8, 114677-114684 (2017).

61. Nduom, E. K. et al. PD-L1 expression and prognostic impact in glioblastoma. Neuro-Oncol. 18, 195-205 (2016). 
62. Liu, J. et al. Mir-758-5p Suppresses Glioblastoma Proliferation, Migration and Invasion by Targeting ZBTB20. Cell. Physiol. Biochem.: Int. J. Exp. Cell. Physiol. Biochem. Pharmacol. 48, 2074-2083 (2018).

63. Parat, M. O. \& Riggins, G. J. Caveolin-1, caveolae, and glioblastoma. NeuroOncol. 14, 679-688 (2012).

64. Nakahata, A. M., Suzuki, D. E., Rodini, C. O., Fiuza, M. L. \& Okamoto, O. K. RNAimediated knockdown of E2F2 inhibits tumorigenicity of human glioblastoma cells. Oncol. Lett. 8, 1487-1491 (2014).

65. Zhu, A. et al. Molecular mechanism of SSFA2 deletion inhibiting cell proliferation and promoting cell apoptosis in glioma. Pathol., Res. Pract. 215, 600-606 (2019).

66. Uchio-Yamada, K. et al. Tenc1-deficient mice develop glomerular disease in a strain-specific manner. Nephron. Exp. Nephrol. 123, 22-33 (2013).

67. Colamaio, M. et al. HMGA1 silencing reduces stemness and temozolomide resistance in glioblastoma stem cells. Expert Opin. Therapeutic Targets $\mathbf{2 0}$ 1169-1179 (2016)
68. Cook, P. J. et al. Cox-2-derived PGE2 induces Id1-dependent radiation resistance and self-renewal in experimental glioblastoma. Neuro-Oncol. 18, 1379-1389 (2016)

69. Jin, X. et al. EGFR-AKT-Smad signaling promotes formation of glioma stem-like cells and tumor angiogenesis by ID3-driven cytokine induction. Cancer Res. 71 7125-7134 (2011).

70. Katsetos, C. D., Draberova, E., Legido, A., Dumontet, C. \& Draber, P. Tubulin targets in the pathobiology and therapy of glioblastoma multiforme. I. Class III beta-tubulin. J. Cell. Physiol. 221, 505-513 (2009).

71. Hsu, Y. C. et al. Activation of Aurora A kinase through the FGF1/FGFR signaling axis sustains the stem cell characteristics of glioblastoma cells. Exp. Cell Res. 344, 153-166 (2016).

72. Meiser, J. et al. Increased formate overflow is a hallmark of oxidative cancer. Nat. Commun. 9, 1368 (2018).

73. Tie, X., Han, S., Meng, L., Wang, Y. \& Wu, A. NFAT1 is highly expressed in, and regulates the invasion of, glioblastoma multiforme cells. PLOS ONE 8, e66008 (2013). 